\title{
INTERACTIVE CADASTRAL BOUNDARY DELINEATION FROM UAV DATA
}

\author{
S. Crommelinck ${ }^{\text {a, }}$, B. Höfle b, c, d, M. N. Koeva a , M. Y. Yang a , G. Vosselman ${ }^{\text {a }}$ \\ ${ }^{a}$ Faculty of Geo-Information Science and Earth Observation (ITC), University of Twente, Enschede, the Netherlands - \\ (s.crommelinck, m.n.koeva, michael.yang, george.vosselman)@utwente.nl \\ ${ }^{\mathrm{b}}$ GIScience Research Group, Institute of Geography, Heidelberg University, Germany - hoefle@ uni-heidelberg.de \\ ${ }^{\mathrm{c}}$ Heidelberg Center for the Environment (HCE), Heidelberg University, Germany \\ d Interdisciplinary Center for Scientific Computing (IWR), Heidelberg University, Germany
}

\section{Commission II, WG II/4}

KEY WORDS: UAV Photogrammetry, Image Analysis, Image Segmentation, Object Detection, Cadastral Boundary Delineation, Machine Learning, Land Administration, Cadastral Mapping

\begin{abstract}
:
Unmanned aerial vehicles (UAV) are evolving as an alternative tool to acquire land tenure data. UAVs can capture geospatial data at high quality and resolution in a cost-effective, transparent and flexible manner, from which visible land parcel boundaries, i.e., cadastral boundaries are delineable. This delineation is to no extent automated, even though physical objects automatically retrievable through image analysis methods mark a large portion of cadastral boundaries. This study proposes (i) a methodology that automatically extracts and processes candidate cadastral boundary features from UAV data, and (ii) a procedure for a subsequent interactive delineation. Part (i) consists of two state-of-the-art computer vision methods, namely gPb contour detection and SLIC superpixels, as well as a classification part assigning costs to each outline according to local boundary knowledge. Part (ii) allows a user-guided delineation by calculating least-cost paths along previously extracted and weighted lines. The approach is tested on visible road outlines in two UAV datasets from Germany. Results show that all roads can be delineated comprehensively. Compared to manual delineation, the number of clicks per $100 \mathrm{~m}$ is reduced by up to $86 \%$, while obtaining a similar localization quality. The approach shows promising results to reduce the effort of manual delineation that is currently employed for indirect (cadastral) surveying.
\end{abstract}

\section{INTRODUCTION}

Unmanned aerial vehicles (UAVs) are rapidly developing and increasingly applied in remote sensing, as they fill the gap between ground based sampling and airborne observations. Numerous application fields make use of the cost-effective, flexible and rapid acquisition system delivering orthoimages, point clouds and digital surface models (DSMs) of high resolution (Colomina and Molina, 2014; Nex and Remondino, 2014).

Recently, the use of UAVs in land administration is expanding (Jazayeri et al., 2014; Koeva et al., 2016; Manyoky et al., 2011; Maurice et al., 2015): the high-resolution imagery is often used to visually detect and manually digitize cadastral boundaries. Such boundaries outline land parcels, for which additional information such as ownership and value are saved in a corresponding register (IAAO, 2015). The resulting cadastral map is considered crucial for a continuous and sustainable recording of land rights, as it allows the establishment of bureaucratic systems of fiscal and juridical nature and facilitates economic decision-making (Williamson et al., 2010).

Worldwide, the land rights of over $70 \%$ of the population are unrecognized, wherefore innovative, affordable, reliable, transparent, scalable and participatory tools for fit-for-purpose and responsible land administration are sought (Enemark et al., 2014). Automatically extracting visible cadastral boundaries from UAV data by providing a publicly available approach to edit and finalize those boundaries would meet this demand and improve current mapping procedures in terms of cost, time and accuracy (Luo et al., 2017).
This study describes advancements in developing a corresponding approach for UAV-based mapping of visible cadastral boundaries. It is based on the assumption that a large portion of cadastral boundaries is manifested through physical objects such as hedges, fences, stone walls, tree lines, roads, walkways or waterways. Those boundaries, visible in the RGB as well as the DSM data, bear the potential to be extracted in part automatically (Zevenbergen and Bennett, 2015). The extracted outlines require (legal) adjudication and incorporation of local knowledge from human operators in order to derive final cadastral boundaries.

In past work, a hypothetical generalized workflow for the automatic extraction of visible cadastral boundaries has been proposed (Crommelinck et al., 2016). It was derived from 89 studies that extract physical objects related to those manifesting cadastral boundaries from high-resolution optical sensor data. The synthesized methodology consists of image segmentation, line extraction and contour generation (Figure 1). For image segmentation, globalized probability of boundary $(\mathrm{gPb})$ contour detection was found to be applicable for an initial detection of visible boundaries. However, the method does not enable the processing of large images. Therefore, the UAV data were reduced in resolution, which led to a reduced localization quality (Crommelinck et al., 2017b). The localization quality at the locations of initially detected candidate boundaries is improved through the proceeding workflow component. For line extraction, simple linear iterative clustering (SLIC) superpixels applied to the full-resolution data were found to coincide largely with object boundaries in terms of completeness and correctness (Crommelinck et al., 2017a).

\footnotetext{
* Corresponding author
} 
The aim of this study is to describe the final workflow component of contour generation: $\mathrm{gPb}$ contour detection and SLIC superpixels are combined with a random forest (RF) classifier and processed in a semi-automatic procedure that allows a subsequent delineation of visible boundaries. Overall, the study contributes to advancements in developing a methodology for UAV-based delineation of visible cadastral boundaries (Sect. 2.3). It uses RGB and DSM information and is designed for rural areas, in which physical objects such as roads are clearly visible and are anticipated to coincide with fixed cadastral boundaries.

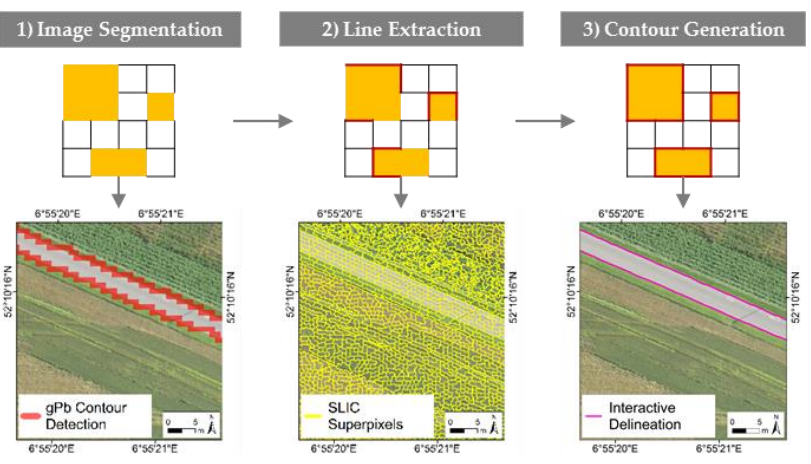

Figure 1. Sequence of a commonly applied workflow proposed in (Crommelinck et al., 2016). It aims to extract physical objects related to those manifesting cadastral boundaries from highresolution optical sensor data. For the first and second component, state-of-the-art computer vision approaches have been evaluated separately and determined as efficient for UAVbased cadastral mapping (Crommelinck et al., 2017a; Crommelinck et al., 2017b). The third component as well as the overall approach is described in this paper.

\section{MATERIALS AND METHODS}

\subsection{UAV Data}

Two rural areas in Amtsvenn and Gerleve in Germany were selected for this study (Table 2, Figure 7). The data were captured with indirect georeferencing, i.e., Ground Control Points (GCPs) were distributed within the field and measured with a Global Navigation Satellite System (GNSS). RGB orthoimages as well as DSMs were generated with Pix4DMapper.

\begin{tabular}{rcc}
\hline & Amtsvenn & Gerleve \\
\hline UAV model & GerMAP G180 & DT18 PPK \\
camera/focal length & Ricoh GR/18.3 & DT-3Bands RGB/12 \\
forw./sidew. overlap [\%] & $80 / 65$ & $80 / 70$ \\
GSD [m] & 0.05 & 0.03 \\
extent [m] & $1000 \times 1000$ & $1000 \times 1000$ \\
\hline
\end{tabular}

Table 2. Specifications of UAV data.

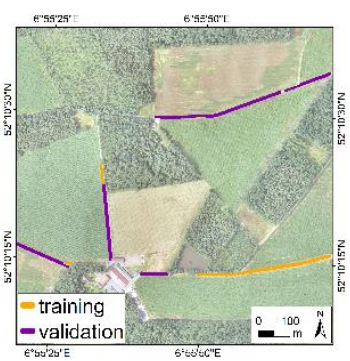

(a)

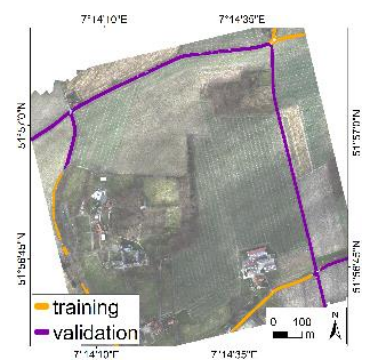

(b)
Figure 7. UAV data from (a) Amtsvenn and (b) Gerleve overlaid with SLIC lines used for training (30\%) and validation (70\%).

\subsection{Image Processing Workflow}

The image processing workflow is based on the one shown in Figure 1. Its three components are described in Sect. 2.2.1 - 2.2.3. and visualized in Figure 5. Corresponding source code, with test data and a step-by-step guide is publically available (Crommelinck, 2017a). The interactive component is implemented in an open source GIS (Crommelinck, 2017b).

\subsubsection{Image Segmentation - gPb Contour Detection:} Contour detection refers to finding closed boundaries between objects or segments. Globalized probability of boundary $(\mathrm{gPb})$ contour detection refers to the processing pipeline visualized in Figure 2, explained in this section and based on (Arbelaez et al., 2011). This pipeline originates from computer vision and aims to find closed boundaries between objects or segments in an image. This is achieved through combining edge detection and hierarchical image segmentation, while integrating image information on texture, color and brightness on both a local and a global scale.

In a first step, oriented gradient operators for brightness, color and texture are calculated on two halves of differently scaled discs to obtain local image information. The cues are merged based on a logistic regression classifier resulting in a posterior probability of a boundary, i.e., an edge strength per pixel. The global image information is obtained through spectral clustering detecting the most salient edges only. This is done by examining a radius of pixels around a target pixel in terms of oriented gradient operators as for the local image information. The local and global information are combined through learning techniques and trained on natural images from the 'Berkeley Segmentation Dataset and Benchmark' (Arbeláez et al., 2007). By considering image information on different scales, relevant boundaries are verified, while irrelevant ones, e.g., in textured regions, are eliminated. This is referred to as global optimization in the following. In the second step, initial regions are formed from the oriented contour signal provided by a contour detector through oriented watershed transformation. Subsequently, a hierarchical segmentation is performed through weighting each boundary and their agglomerative clustering to create an ultrametric contour map (ucm) that defines the hierarchical segmentation.

The overall result consists of (i) a contour map, in which each pixel is assigned a probability of being a boundary pixel, and (ii) a binary boundary map containing closed contours, in which each pixel is labeled as 'boundary' or 'no boundary'. The approach has been shown to be applicable to UAV orthoimages for an initial localization of candidate object boundaries (Crommelinck et al., 2017b). UAV orthoimages of extents larger than $1000 \times 1000$ pixels need to be reduced in resolution, due to the global optimization of the original implementation. The localization quality of initially detected candidate boundaries is improved through the following workflow components that use the full-resolution RGB and DSM data.

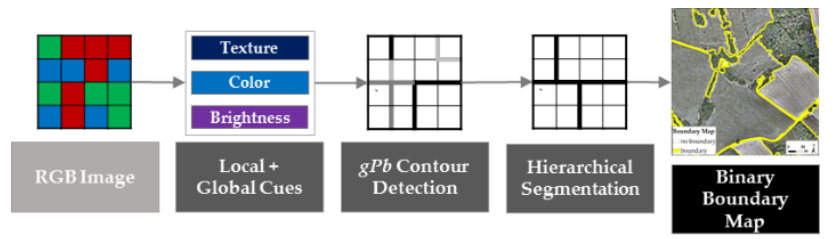

Figure 2. Processing pipeline of globalized probability of boundary $(\mathrm{gPb})$ contour detection and hierarchical image segmentation resulting in a binary boundary map containing closed boundaries. 

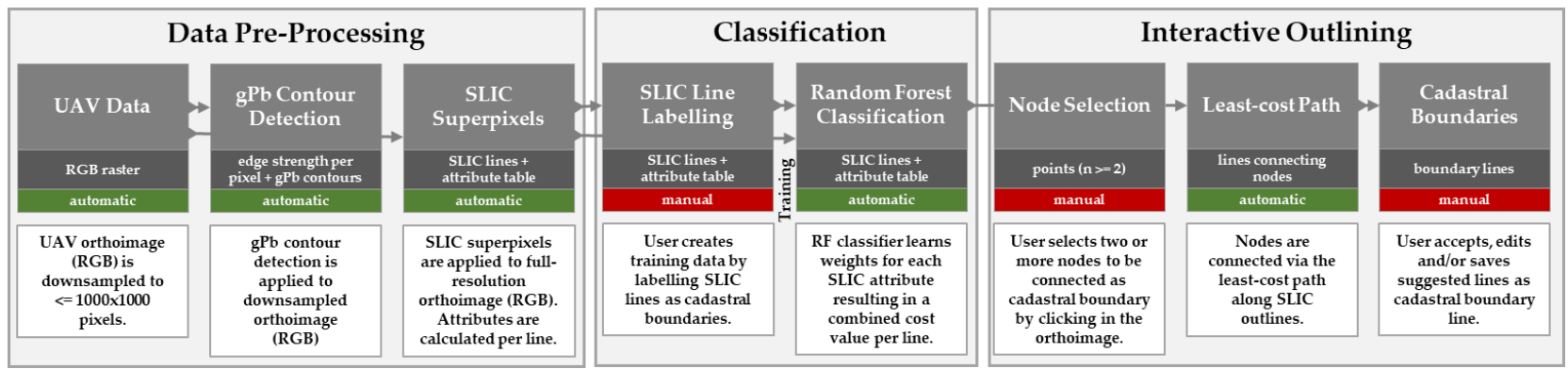

Figure 5. Delineation workflow combining the methods described in Sect. 2.2.1 - 2.2.3.

2.2.2 Line Extraction - SLIC Superpixels: Simple linear iterative clustering (SLIC) superpixels originate from computer vision and are introduced in (Ren and Malik, 2003). Superpixels aim to group pixels into perceptually meaningful atomic regions and can therefore be located between pixel- and object-based approaches. The approach allows to compute image features for each superpixel rather than each pixel, which reduces subsequent processing tasks in complexity and computing time. Further, the boundaries of superpixels adhere well to object outlines in the image and can therefore be used to delineate objects (Neubert and Protzel, 2012).

When comparing state-of-the-art superpixel approaches, SLIC superpixels have outperformed comparable approaches in terms of speed, memory efficiency, compactness and correctness of outlines (Csillik, 2016; Schick et al., 2012; Stutz et al., 2017). The approach, visualized in Figure 3, was introduced and extended by Achanta el al. (2010, 2012). SLIC considers image pixels in a $5 \mathrm{D}$ space, in terms of their $\mathrm{L} * \mathrm{a} * \mathrm{~b}$ values of the CIELAB color space and their $\mathrm{x}$ and $\mathrm{y}$ coordinates. Subsequently, the pixels are clustered based on an adapted k-means clustering. The clustering considers color similarity and spatial proximity. SLIC implementations are widely available. This study applies the GRASS implementation (Kanavath and Metz, 2017).

The approach has been shown to be applicable to UAV orthoimages of $0.05 \mathrm{~m}$ ground sample distance (GSD) (Crommelinck et al., 2017a). Further, cadastral boundaries demarcated through physical objects often coincide with the outlines of SLIC superpixels.

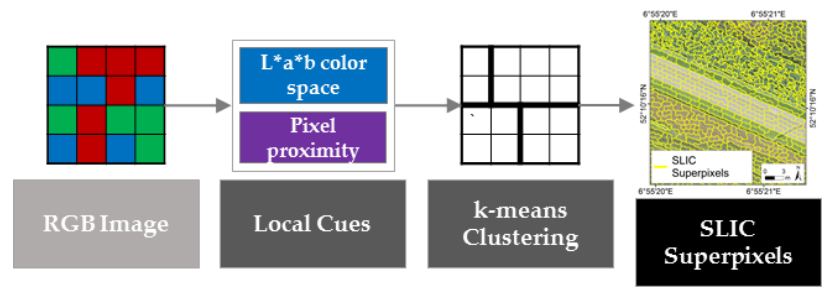

Figure 3. Processing pipeline of simple linear iterative clustering (SLIC) resulting in agglomerated groups of pixels, i.e., superpixels, whose boundaries outline physical objects in the image.

\subsubsection{Contour Generation - Interactive Delineation:} Contour generation refers to generating a vectorized and topologically connected network of SLIC outlines from Sect. 2.2.2 that surround candidate regions from Sect. 2.2.1. This component combines the detection quality of $\mathrm{gPb}$ contour detection with the localization quality of SLIC superpixels. This is realized by seeking a subset of superpixels whose collective boundaries correspond to contours of physical objects in the image.
Levinshtein et al. (2012) first reformulated the problem of finding contour closure to identifying subsets of superpixels that align with physical object contours. The authors combine features such as distance, strength, curvature and alignment to identify edges for image segmentation. These features are combined by learning the best generic weights for their combination on a computer vision benchmark dataset. This approach can be related to perceptual grouping in which local attributes in relation to each other are grouped to form a more informative attribute containing context information (Sowmya and Trinder, 2000). By iteratively grouping low-level image descriptions, a higher-level structure of higher informative value is obtained (Iqbal and Aggarwal, 2002). Perceptual grouping for contour closure is widely applied in computer vision (Estrada and Jepson, 2004; Stahl and Wang, 2007), pattern recognition (Iqbal and Aggarwal, 2002) as well as in remote sensing (Turker and Kok, 2013; Yang and Wang, 2007). The criteria for perceptual grouping are mostly based on the classical Gestalt cues of proximity, continuity, similarity, closure, symmetry, common regions and connectedness that originate from Lowe's early work on perceptual grouping, in which a computational model for parallelism, collinearity, and proximity is introduced (Lowe, 1985). The attributes are mostly combined into a cost function that models the perceptual saliency of the resulting structure.

These ideas are transferable to this study: Wegner et al. (2015) extract road networks from aerial imagery and elevation data by applying superpixel-based image segmentation, classifying the segments with a RF classifier and searching for the Dijkstra leastcost path between segments with high likelihoods of being roads. Warnke and Bulatov (2017) extend this approach by optimizing the methodology in terms of feature selection. They investigate the training step by evaluating two classifiers and show that choosing features largely influences classification quality and that feature importance depends on the selected classifier. Similarly, García-Pedrero et al. (2017) use superpixels as minimum processing units, which is followed by a classificationbased agglomerating of superpixels to obtain a final segmentation of agricultural fields from satellite imagery. All these approaches consider superpixels as segments, i.e., superpixels are agglomerated by comparing features per segment in relation to its adjacent neighbors (García-Pedrero et al., 2017; Santana et al., 2017; Yang and Rosenhahn, 2016), sometimes in combination with boundary information (Jiang et al., 2013; Wang et al., 2017).

In this paper, the problem of finding adjacent superpixels belonging to one object is reformulated to finding parts of superpixel outlines that delineate one object: attributes are not calculated per superpixel, but per outline segment (Figure 4). They are created by splitting each superpixel outline, wherever outlines of three or more adjacent superpixels have a point in common. 19 attributes taking into account the full-resolution RGB and DSM, as well as the low-resolution gPb information are calculated per line (Table 1). Similar to the classical Gestalt 
cues, the attributes consider the SLIC lines themselves (i.e., their geometry) and their spatial context (i.e., their relation to $\mathrm{gPb}$ lines or to underlying RGB and DSM rasters).

\begin{tabular}{|c|c|}
\hline Feature & Description \\
\hline length $[\mathrm{m}]$ & length per SLIC segment along the line \\
\hline ucm_rgb & $\begin{array}{l}\text { median of all ucm_rgb pixels within a } 0.4 \mathrm{~m} \text { buffer } \\
\text { around each SLIC segment }\end{array}$ \\
\hline lap_dsm & $\begin{array}{l}\text { median of all DSM laplacian filter values within a } \\
0.4 \mathrm{~m} \text { buffer around each SLIC segment }\end{array}$ \\
\hline $\begin{array}{r}\text { dist_to_gPb } \\
{[\mathrm{m}]}\end{array}$ & $\begin{array}{l}\text { distance between SLIC segment and } \mathrm{gPb} \text { lines } \\
\text { (overall shortest distance) }\end{array}$ \\
\hline azimuth $\left[{ }^{\circ}\right]$ & $\begin{array}{l}\text { horizontal angle measured clockwise from north per } \\
\text { SLIC segment }\end{array}$ \\
\hline sinuosity & $\begin{array}{l}\text { ratio of distance between start and end point along } \\
\text { SLIC segment (line length) and their direct } \\
\text { Euclidean distance }\end{array}$ \\
\hline azi_gPb $\left[{ }^{\circ}\right]$ & $\begin{array}{l}\text { horizontal angle measured clockwise from north per } \\
\text { gPb segment closest to a SLIC segment (aims } \\
\text { to indicate line parallelism/collinearity) }\end{array}$ \\
\hline r_dsm_medi & $\begin{array}{l}\text { median of all DSM values lying within a } 0.2 \mathrm{~m} \text { buffer } \\
\text { right of each SLIC segment. }\end{array}$ \\
\hline 1_dsm_medi & $\begin{array}{l}\text { median of all DSM values lying within a } 0.2 \mathrm{~m} \text { buffer } \\
\text { left of each SLIC segment }\end{array}$ \\
\hline r_red_medi & $\begin{array}{l}\text { median of all red values lying within a } 0.2 \mathrm{~m} \text { buffer } \\
\text { right of each SLIC segment }\end{array}$ \\
\hline 1_red_medi & $\begin{array}{l}\text { median of all red values lying within a } 0.2 \mathrm{~m} \text { buffer } \\
\text { left of each SLIC segment }\end{array}$ \\
\hline r_gre_medi & $\begin{array}{l}\text { median of all green values lying within a } 0.2 \mathrm{~m} \text { buffer } \\
\text { right of each SLIC segment }\end{array}$ \\
\hline 1_gre_medi & $\begin{array}{l}\text { median of all green values lying within a } 0.2 \mathrm{~m} \text { buffer } \\
\text { left of each SLIC segment }\end{array}$ \\
\hline r_blu_medi & $\begin{array}{l}\text { median of all blue values lying within a } 0.2 \mathrm{~m} \text { buffer } \\
\text { right of each SLIC segment }\end{array}$ \\
\hline 1_blu_medi & $\begin{array}{l}\text { median of all blue values lying within a } 0.2 \mathrm{~m} \text { buffer } \\
\text { left of each SLIC segment }\end{array}$ \\
\hline red_grad & $\begin{array}{l}\text { absolute value of difference between } r \text { red_medi } \\
\text { and 1_red_medi }\end{array}$ \\
\hline green_grad & $\begin{array}{l}\text { absolute value of difference between r_green_medi } \\
\text { and 1_green_medi }\end{array}$ \\
\hline blue_grad & $\begin{array}{l}\text { absolute value of difference between r_blue_medi } \\
\text { and 1_blue_medi }\end{array}$ \\
\hline dsm_grad & $\begin{array}{l}\text { absolute value of difference between } r \_d s m \_ \text {medi } \\
\text { and } 1 \_d s m \_m e d i\end{array}$ \\
\hline
\end{tabular}

For training and validation, one attribute is added manually by labelling SLIC lines corresponding to reference object outlines as 'boundary' or 'no boundary', respectively. The data are divided into $30 \%$ for training and $70 \%$ for validation. The features shown in Table 1 together with the label 'boundary' or 'no boundary' are provided to the RF classifier to learn the combination of features leading to the class 'boundary' for the training data. The trained classifier then uses the features to predict for each line in the validation data a likelihood for each line for belonging to the class 'boundary'. This boundary likelihood $b$ is transformed to a cost value $c$ as shown in the following:

$$
c[0 ; 1]=1-b
$$

where $\quad c=$ cost value per SLIC line

$b=$ boundary likelihood per SLIC line

This cost value $c$ in range $[0 ; 1]$ is used to find the least-cost path between points indicated by a user. The Steiner least-cost path searches for the path along the SLIC lines having the lowest $c$, i.e., the highest likelihood for belonging to the class 'boundary'. The points represent start-, end, and optionally middle-points of a boundary to be delineated. Finally, the result is displayed to the user providing the options to accept, smooth, edit and/or save the line. Smoothing is done using the Douglas-Peucker line simplification. This interactive component is implemented as an open source QGIS plugin (Crommelinck, 2017b).

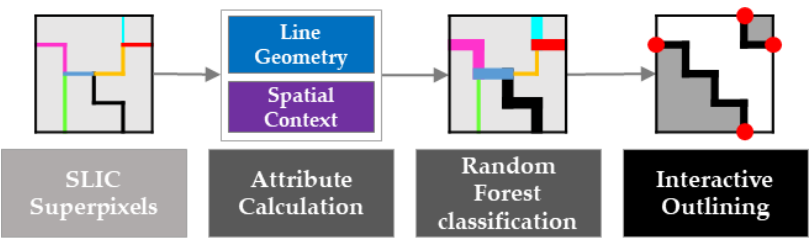

Figure 4. Processing pipeline of interactive delineation: each superpixel outline is split, wherever outlines of three or more adjacent superpixels have a point in common (visualized by line color). Attributes are calculated per line. They are used by a RF classifier to predict boundary likelihoods (visualized by line thickness). User-selected nodes (red points) are connected along the lines of highest likelihoods.

\subsection{Accuracy Assessment}

The methodology is designed and implemented for rural areas, in which the number of visible cadastral boundaries is expected to be higher than in urban ones. As stated above, numerous physical objects can manifest cadastral boundaries. For accuracy assessment in a metric sense, an object was sought, whose outline is clearly delineable. Further, automating the delineation process saves most time for large parcels with long and curved outlines. Luo et al. (2017) have shown that up to $49 \%$ of cadastral boundaries are demarcated by roads and conclude that deriving road outlines would therefore contribute significantly to generating cadastral boundaries. Consequently, roads are selected for accuracy assessment.

The approach is investigated in terms of the components shown in Figure 5. Since the first one, i.e., 'data pre-processing', has been evaluated in previous studies (Crommelinck et al., 2017a; Crommelinck et al., 2017b), the accuracy assessment focuses on 'classification' and the 'interactive outlining'. The accepted accuracy for cadastral boundary surveying depends on local requirements, regulations and the accuracy of the boundaries themselves. Recommendations from the IAAO (2015) range from $0.3 \mathrm{~m}$ for urban areas to $2.4 \mathrm{~m}$ in rural areas for horizontal accuracy. They advise to use these measures judiciously and remain unclear whether this is a maximum for the accepted error or a standard deviation. According to Stock (1998) landowners require a higher accuracy $(0.2 \mathrm{~m})$ than authorities $(0.5 \mathrm{~m})$ for rural boundaries. Details on how this accuracy is measured are not provided.

In this study, which is implemented in rural areas, the accepted accuracy is set to $0.2 \mathrm{~m}$ as maximum distance between delineation and reference data. Reference data are created through manually digitizing visible outlines of roads. Only those visible outlines whose fuzziness did not exceed the accepted accuracy are delineated as reference data.

2.3.1 Classification Performance: How well the RF classifier assigns optimal costs to each SLIC line is crucial for the subsequent least-cost path generation. The performance is investigated by considering the feature importance obtained after applying the trained classifier on the validation dataset, as well as the confusion matrix and the derived correctness (Eq. 2) for different cost values $c$. Due to the analysis according to $c$, completeness is not considered: for larger $c$, more lines are detected, which makes the number of false negatives (FN) and thereby completeness not directly comparable across groups of different $c$. 


$$
\text { correctness }[0 ; 100]=\frac{\mathrm{TP}}{\mathrm{TP}+\mathrm{FP}}
$$

where $\quad \mathrm{TP}=$ true positives

$\mathrm{FP}=$ false positives

The detection quality (Figure 6a) determines, how comprehensively SLIC lines are detected by the RF classifier. This is done by calculating a buffer of radius $0.2 \mathrm{~m}$ around the reference lines. The buffer size is chosen in accordance to the predefined accepted accuracy. SLIC lines are buffered with the smallest radius possible of $0.05 \mathrm{~m}$ in accordance to the GSD of the UAV data. SLIC lines are grouped according to boundary likelihoods $b$, transformed to a cost value $c(2)$ in range $[0 ; 1]$ at increments of 0.2. Each group is overlaid with the buffered reference data to calculate a confusion matrix and a correctness.

The localization quality (Figure 6b) determines, if low $c$ are assigned to segments located closer to the reference data. This is done by buffering the reference data with radii of $0.05,0.1,0.15$, and $0.2 \mathrm{~m}$. The previously buffered and grouped SLIC lines are reused. Each group is overlaid with the buffered reference data to generate a confusion matrix and to calculate the sum of TP pixels per buffer distance.

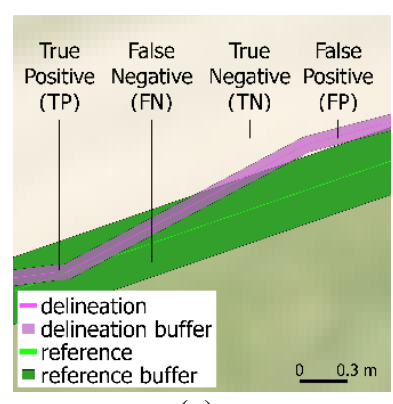

(a)

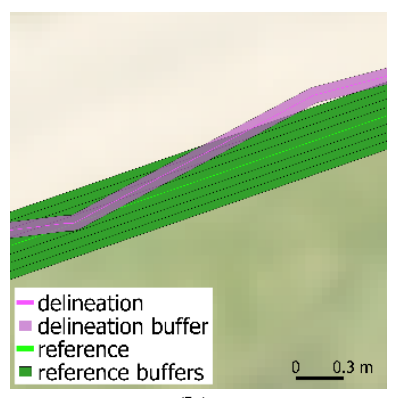

(b)
Figure 6. (a) Detection quality, for which delineation data are buffered with $0.05 \mathrm{~m}$ and reference data with $0.2 \mathrm{~m}$. Both are overlaid to calculate the number of pixels being TP, FN, TN or FP. (b) Localization quality, for which the reference data are buffered with $0.05-0.2 \mathrm{~m}$ and overlaid with the buffered delineation data to calculate the sum of TPs per buffer distance.

2.3.2 Interactive Outlining Performance: If and to what extent the interactive delineation is superior to manual delineation is the focus of this section. This is done by defining a user scenario and delineating all visible road outlines once manually, once interactively. Metric accuracy measures are calculated for both datasets. The user scenario encompasses the guideline of using as few clicks as necessary to delineate all visible roads within the accepted accuracy of $0.2 \mathrm{~m}$. The metric accuracy measures consist of the calculation of the localization quality as described above and the average number of required clicks per $100 \mathrm{~m}$

\section{RESULTS}

The results reveal that the assignment of $c$ works as desired: road outlines are comprehensively covered by SLIC lines of low $c$ values and the correctness decreases for higher $c$ (Table 3). Similarly, the localisation quality mostly decreases for higher $c$ ,i.e., the classifier assigs low $c$ values for a high percentage of lines close to the reference data (Figure 8 ). These values would vary when changing the buffer size or taking into account different lines for training.

The calculated feature importance for features shown in Table 1 reveals that higher-order features are often more valuable, i.e., a feature containing the gradient between green values right and left of the SLIC line (green_grad) is more important than a feature containing averaged green values underlying a SLIC line (1_gre_medi, r_gre_medi). DSM-related features have low importance (dsm_grad, lap_dsm, r_dsm_medi, 1_dsm_medi), which can be increased by considering another physical object, whose outlines are stronger demarcated through height difference and by using relative height as a feature. gPb-related features (ucm_rgb, dist_to_gPb, azi_gPb) have a low importance, which might be caused by the low resolution of the $\mathrm{gPb}$ data. Tiling does not solve this problem, since the global optimization requires image information on a global scale. However, gPb contours are still relevant as they are used to narrow down the area of investigation and thus reduce processing time. The results give an initial estimation of feature importance, but would require more data to analysable in depth.

\begin{tabular}{rccc}
\hline & & Amtsvenn & Gerleve \\
\hline & SLIC line segments $(\mathrm{N})$ & 22,183 & 57,500 \\
SLIC line segments [m] & 37,063 & 72,333 \\
\hline \multirow{2}{*}{ correctness $(c=0.0-0.19)[\%]$} & 86 & 93 \\
& correctness $(c=0.2-0.39)[\%]$ & 90 & 96 \\
correctness $(c=0.4-0.59)[\%]$ & 78 & 88 \\
& correctness $(c=0.6-0.79)[\%]$ & 61 & 70 \\
\hline
\end{tabular}

Table 3. Classification performance: detection quality for SLIC lines of different cost value $c$ compared to reference data.

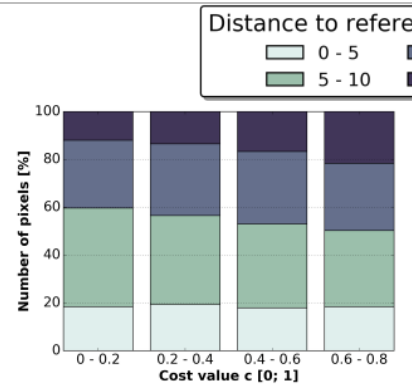

(a)

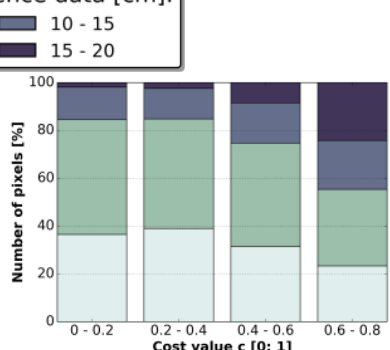

(b)
Figure 8. Classification performance: localization quality for SLIC lines of different cost values $c$ assigned through the RF classification for (a) Amtsvenn and (b) Gerleve.

The interactive outlining performance visualized in Figure 9 reveals that road outlines are successfully demarcated by low $c$ values generated through RF classification (a). The interactive delineation visualized in (b) allows to select nodes (yellow) from a set of nodes (red), that are automatically connected (green) along the SLIC lines of least cost. The interactive delineation saves most clicks, when delineating long and curved roads as shown in (c), where the interactive delineation of a line of $274 \mathrm{~m}$ length requires two clicks only. For road parts covered by vegetation or those having narrow or fuzzy boundaries manual delineation is superior (d). High gradients inside a road can cause the least-cost path to run along the middle of the road, which can be avoided by placing an additional node (yellow) along the road outline (e, f). The least-cost path favors less segments of high costs over more segments of lower costs, since the summated costs of the entire path are considered (g). Created outlines can be smoothed out through the build-in line simplification that transforms the initial least-cost path (blue) to a simpler path (green) (h). 
In general, all visible road outlines were delineated. For Amtsvenn $0 \%$ and for Gerleve 5\% of lines required minor editing, in cases, where SLIC outlines do not run along the desired road outline (Figure 9d). The localization quality (Figure 10) visualizes the portion of delineated lines located at different distances to the reference data. It shows that for Amtsvenn almost $60 \%$ and for Gerleve almost $80 \%$ of boundaries delineated with the interactive approach are within $10 \mathrm{~cm}$ of the reference data. These results together with the decrease of required clicks, i.e., a reduction by $86 \%$ for Amtsvenn and $76 \%$ for Gerleve (Table 4), and the lower zoom level required for delineation, shows that the interactive delineation is superior in terms of effort to delineate visible roads from UAV data.

\begin{tabular}{rcccc}
\hline & \multicolumn{2}{c}{ Amtsvenn } & \multicolumn{2}{c}{ Gerleve } \\
& manual & interact. & manual & interact. \\
\hline line segments [m] & 1,900 & 1,915 & 3,911 & 3,922 \\
avg. clicks per & 14.2 & 2.3 & 21.2 & 4.5 \\
$100 \mathrm{~m}(\mathrm{~N})$ & $(100 \%)$ & $(86 \%)$ & $(100 \%)$ & $(76 \%)$ \\
\hline
\end{tabular}

Table 4. Interactive outlining performance: general statistics for the manual and the interactive delineation.

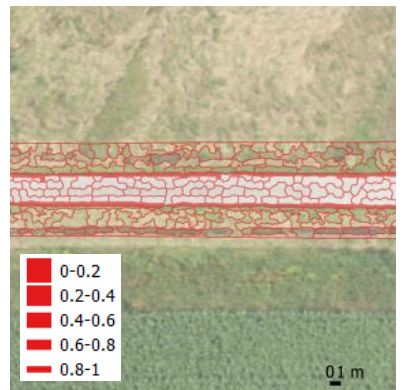

(a)

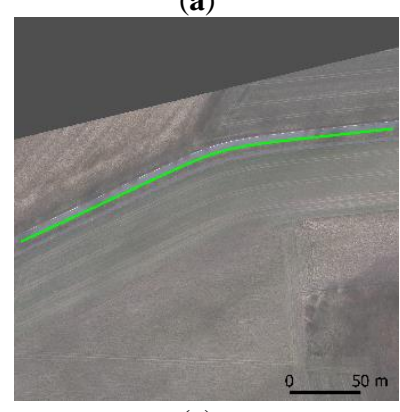

(c)

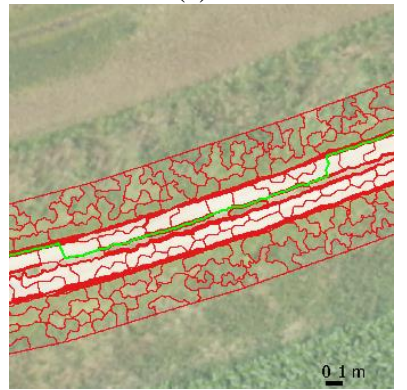

(e)

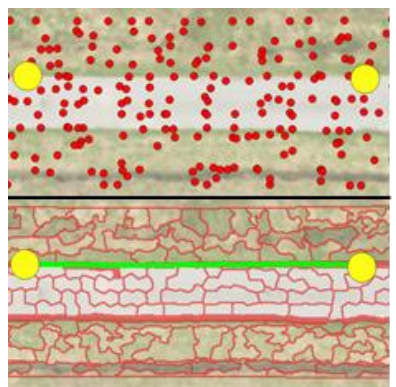

(b)

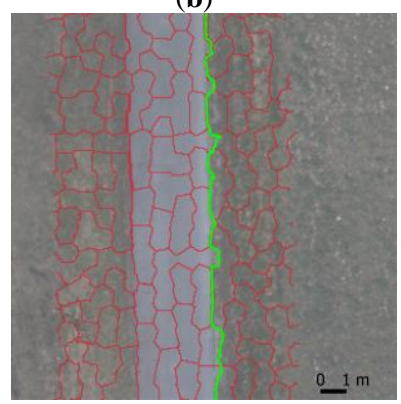

(d)

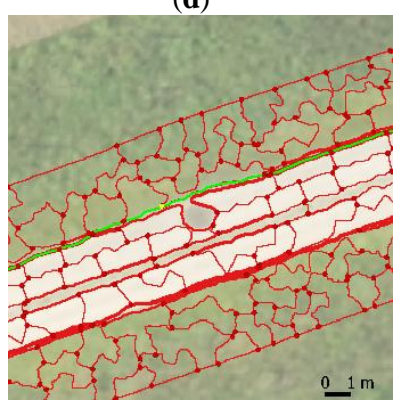

(f)

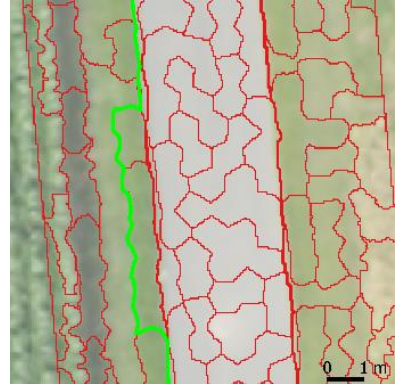

(g)

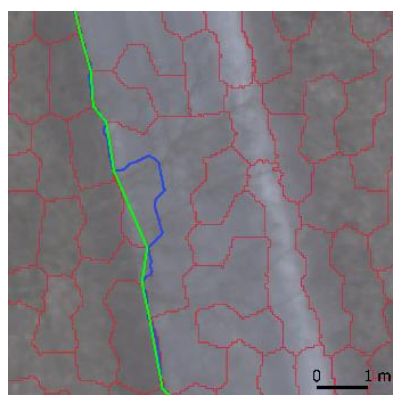

(h)
Figure 9. Examples of the interactive delineation (green) along SLIC lines (red). The thicker a SLIC line, the lower $c$.

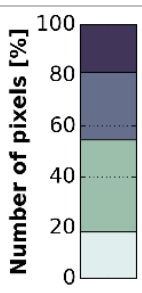

(a)
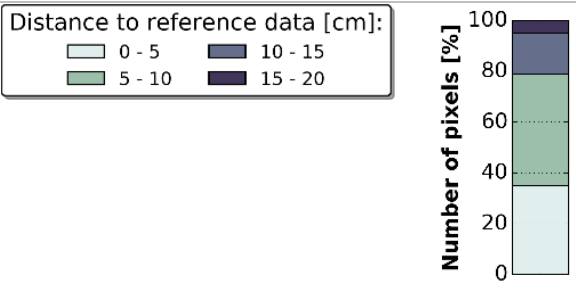

(b)
Figure 10. Interactive outlining performance: localization quality for delineation for (a) Amtsvenn and (b) Gerleve. Both the reference and the interactively delineated data consists of lines that are rasterized to quantify the localization quality.

\section{DISCUSSION}

In general, the methodology could improve current indirect mapping procedures by making them more reproducible and efficient. However, a certain skill level of the surveyors in geodata processing is required as well as the presence of visible cadastral boundaries. With cadastral boundaries being a human construct, certain boundaries are not automatically detectable, wherefore semi-automatic approaches are required (Luo et al., 2017).

Limitations of the accuracy assessment are as follows: labelled training data doesn't always coincide exactly with the reference data, as SLIC outlines do not perfectly match the manually delineated road outlines. Furthermore, some roads have fuzzy outlines, wherefore a certain outline is selected within the accepted accuracy for both the manual and the interactive delineation. Furthermore, manual image interpretation is prone to produce ambiguous results due to interpreters generalizing differently. These uncertainties propagate through the accuracy measures and would increase when considering physical objects of fuzzier outlines (Albrecht, 2010; García-Pedrero et al., 2017). Further, the percentage of roads demarcating cadastral boundaries, which according to Luo et al. (2017) amounts up to $49 \%$ might be lower in certain cases. Further work should be conducted considering various objects in relation to real cadastral reference data.

Future work could focus on identifying optimal features for classification (Genuer et al., 2010; Warnke and Bulatov, 2017). The optimal selection of training data could be supported by active learning strategies. Another focus would be to extent the approach to different physical objects, datasets and scenarios by developing a classifier transferable across scenes. However, even manually labelling $30 \%$ of the data before being able to apply the interactive delineation as done in this study, would still be superior in terms of effort than delineating $100 \%$ manually. Existing cadastral data might be used to automatically generate 
training data. The transferability to data from aerial or satellite platforms could be considered to determine the degree to which high-resolution UVA data containing detailed 3D information is beneficial or required for indirect cadastral surveying. Further, the least-cost paths generation can be improved by scaling the line costs with their length to avoid the path favouring few segments of high cost over many segments of low costs (Figure $9 \mathrm{~g}$ ). In addition, sharp edges in the generated least-cost path can be penalized to reduce outlier occurrence, as done in snake approaches.

\section{CONCLUSION}

This study contributes to developing a methodology for UAVbased delineation of visible cadastral boundaries. This is done by proposing a methodology that partially automates and simplifies the delineation of outlines of physical objects such as roads demarcating cadastral boundaries. Previous work has focused on automatically extracting RGB image information for that methodology. In this paper, the methodology is extended by a classification and an interactive outlining part applied to RGB and DSM data. Furthermore, this study proposes a methodology to automate cadastral mapping covering all required steps after obtaining UAV data to generating candidate cadastral boundary lines.

The reformulated problem of delineating physical objects from image data to combining line feature information with RF classification presented in this study, could be beneficial for different delineation applications. The aim of this study is to apply the suggested approach for cadastral mapping. In this field, the approach has shown promising results to reduce the effort of current indirect surveying approach based on manual delineation. Highest savings are obtained for long and curved outlines. Future work will focus on the methodology's transferability to real world cadastral mapping scenarios.

\section{ACKNOWLEDGMENTS}

This work was supported by its4land, which is part of the Horizon 2020 program of the European Union (project number 687828). We are grateful to Claudia Stöcker for capturing and processing the UAV data, as well as to the GIScience Research Group at Heidelberg University for supporting this work during a 6-month research visit.

\section{REFERENCES}

Achanta, R., Shaji, A., Smith, K., Lucchi, A., Fua, P., Susstrunk, S., 2012. SLIC superpixels compared to state-of-the-art superpixel methods. IEEE Transactions on Pattern Analysis and Machine Intelligence, 34(11), pp. 2274-2282.

Achanta, R., Shaji, A., Smith, K., Lucchi, A., Fua, P., Süsstrunk, S., 2010. SLIC superpixels, EPFL Technical Report no. 149300.

Albrecht, F., 2010. Uncertainty in image interpretation as reference for accuracy assessment in object-based image analysis, Porc. 9th Int. Symp. on Spatial Accuracy Assessment in Natural Resources and Environmental Sciences, pp. 13-16.

Arbeláez, P., Fowlkes, C., Martin, D., 2007. Berkeley segmentation dataset and benchmark, https://www2.eecs.berkeley.edu/Research/Projects/CS/vision/bs ds/ (10 Nov. 2016).
Arbelaez, P., Maire, M., Fowlkes, C., Malik, J., 2011. Contour detection and hierarchical image segmentation. Pattern Analysis and Machine Intelligence, 33(5), pp. 898-916.

Colomina, I., Molina, P., 2014. Unmanned aerial systems for photogrammetry and remote sensing: a review. ISPRS Journal of Photogrammetry and Remote Sensing, 92, pp. 79-97.

Crommelinck, S., 2017a. Delineation-Tool GitHub, https://github.com/SCrommelinck/Delineation-Tool (14 Dec. 2017).

Crommelinck, S., 2017b. QGIS Python Plugins Repository: BoundaryDelineation,

http://plugins.qgis.org/plugins/BoundaryDelineation/ (13 June 2017).

Crommelinck, S., Bennett, R., Gerke, M., Koeva, M., Yang, M.Y., Vosselman, G. SLIC superpixels for object delineation from UAV data. In: International Conference on Unmanned Aerial Vehicles in Geomatics, Bonn, Germany, 04-07 September, pp. 9-16.

Crommelinck, S., Bennett, R., Gerke, M., Nex, F., Yang, M.Y., Vosselman, G., 2016. Review of automatic feature extraction from high-resolution optical sensor data for UAV-based cadastral mapping. Remote Sensing, 8(8), pp. 1-28.

Crommelinck, S., Bennett, R., Gerke, M., Yang, M.Y., Vosselman, G., 2017b. Contour detection for UAV-based cadastral mapping. Remote Sensing, 9(2), pp. 1-13.

Csillik, O. Superpixels: the end of pixels in OBIA. A comparison of stat-of-the-art superpixel methods for remote sensing data. In: GEOBIA, Enschede, the Netherlands, 14-16 September, pp. 1-5.

Enemark, S., Bell, K.C., Lemmen, C., McLaren, R., 2014. Fitfor-purpose land administration. Int. Federation of Surveyors, Frederiksberg, Denmark.

Estrada, F.J., Jepson, A.D., 2004. Perceptual grouping for contour extraction, Proc. 17th Int. Conf. on Pattern Recognition (ICPR), pp. 32-35.

García-Pedrero, A., Gonzalo-Martín, C., Lillo-Saavedra, M. 2017. A machine learning approach for agricultural parcel delineation through agglomerative segmentation. Int. Journal of Remote Sensing, 38(7), pp. 1809-1819.

Genuer, R., Poggi, J.-M., Tuleau-Malot, C., 2010. Variable selection using random forests. Pattern Recognition Letters, 31(14), pp. 2225-2236.

IAAO, 2015. Standard on digital cadastral maps and parcel identifiers. Int. Association of Assessing Officers (IAAO), Kansas City, MO, USA.

Iqbal, Q., Aggarwal, J.K., 2002. Retrieval by classification of images containing large manmade objects using perceptual grouping. Pattern Recognition, 35(7), pp. 1463-1479.

Jazayeri, I., Rajabifard, A., Kalantari, M., 2014. A geometric and semantic evaluation of 3D data sourcing methods for land and property information. Land Use Policy, 36, pp. 219-230. 
Jiang, H., Wu, Y., Yuan, Z. Probabilistic salient object contour detection based on superpixels. In: Conf. on Image Processing (ICIP), Melbourne, Australia, 15-18 September, pp. 3069-3072.

Kanavath, R., Metz, M., 2017. GRASS SLIC superpixels, https://grass.osgeo.org/grass72/manuals/addons/i.superpixels.sli c.html (4 Dec. 2017).

Koeva, M., Muneza, M., Gevaert, C., Gerke, M., Nex, F., 2016. Using UAVs for map creation and updating. A case study in Rwanda. Survey Review, pp. 1-14.

Levinshtein, A., Sminchisescu, C., Dickinson, S., 2012. Optimal image and video closure by superpixel grouping. Int. Journal of Computer Vision, 100(1), pp. 99-119.

Lowe, D., 1985. Perceptual organization and visual recognition. Springer.

Luo, X., Bennett, R., Koeva, M., Lemmen, C., Quadros, N., 2017. Quantifying the overlap between cadastral and visual boundaries: a case study from Vanuatu. Urban Science, 1(4), pp. 32.

Manyoky, M., Theiler, P., Steudler, D., Eisenbeiss, H. Unmanned aerial vehicle in cadastral applications. In: Int. Archives of the Photogrammetry, Remote Sensing and Spatial Information Sciences, Zurich, Switzerland, 14-16 September, pp. 1-6.

Maurice, M.J., Koeva, M.N., Gerke, M., Nex, F., Gevaert, C. A photogrammetric approach for map updating using UAV in Rwanda. In: GeoTechRwanda, Kigali, Rwanda, 18-20 November, pp. 1-8.

Neubert, P., Protzel, P., 2012. Superpixel benchmark and comparison, Proc. Forum Bildverarbeitung, Regensburg, Germany, pp. 1-12.

Nex, F., Remondino, F., 2014. UAV for 3D mapping applications: a review. Applied Geomatics, 6(1), pp. 1-15.

Ren, X., Malik, J. Learning a classification model for segmentation. In: Int. Conf. on Computer Vision (ICCV), Washington, DC, US, 13-16 October, pp. 10-17.

Santana, T.M.H.C., Machado, A.M.C., de A. Araújo, A., dos Santos, J.A. Star: a contextual description of superpixels for remote sensing image classification. In: Progress in Pattern Recognition, Image Analysis, Computer Vision, and Applications, Lima, Peru, 8-11 November, pp. 300-308.

Schick, A., Fischer, M., Stiefelhagen, R. Measuring and evaluating the compactness of superpixels. In: Int. Conf. on
Pattern Recognition (ICPR), Tsukuba Science City, Japan, 11-15 November, pp. 930-934.

Sowmya, A., Trinder, J., 2000. Modelling and representation issues in automated feature extraction from aerial and satellite images. ISPRS Journal of Photogrammetry and Remote Sensing, 55(1), pp. 34-47.

Stahl, J.S., Wang, S., 2007. Edge grouping combining boundary and region information. IEEE Transactions on Image Processing, 16(10), pp. 2590-2606.

Stock, K.M., 1998. Accuracy requirements for rural land parcel boundaries. Australian Surveyor, 43(3), pp. 165-171.

Stutz, D., Hermans, A., Leibe, B., 2017. Superpixels: an evaluation of the state-of-the-art. Computer Vision and Image Understanding, pp. 1-32.

Turker, M., Kok, E.H., 2013. Field-based sub-boundary extraction from remote sensing imagery using perceptual grouping. ISPRS Journal of Photogrammetry and Remote Sensing, 79, pp. 106-121.

Wang, X.-Y., Wu, C.-W., Xiang, K., Chen, W., 2017. Efficient local and global contour detection based on superpixels. Journal of Visual Communication and Image Representation, 48, pp. 77 87.

Warnke, S., Bulatov, D. Variable selection for road segmentation in aerial images. In: Int. Archives of the Photogrammetry, Remote Sensing and Spatial Information Sciences, Hannover, Germany, 6-9 June, pp. 297-304.

Williamson, I., Enemark, S., Wallace, J., Rajabifard, A., 2010. Land administration for sustainable development. ESRI Press Academic, Redlands, CA, USA.

Yang, J., Wang, R., 2007. Classified road detection from satellite images based on perceptual organization. Int. Journal of Remote Sensing, 28(20), pp. 4653-4669.

Yang, M.Y., Rosenhahn, B., 2016. Superpixel cut for figureground image segmentation. ISPRS Annals of the Photogrammetry, Remote Sensing and Spatial Information Sciences, III-3, pp. 387-394.

Zevenbergen, J., Bennett, R. The visible boundary: more than just a line between coordinates. In: GeoTechRwanda, Kigali, Rwanda, 18-20 November, pp. 1-4. 